\title{
Preparation and Photocatalytic Application of Terbium and Sulfur Co- doped Titanium Nanomaterials
}

\author{
Zhencui Wang ( $\nabla$ wzhencui2008@126.com) \\ Hainan Medical University \\ Yuanjiao Wang \\ Hainan Medical University \\ Tianle Tang \\ Hainan Medical University
}

\section{Research Article}

Keywords: Dye wastewater, Tb, S co-doped TiO2, Synergistic effect, Photocatalytic application.

Posted Date: January 25th, 2022

DOI: https://doi.org/10.21203/rs.3.rs-1198538/v1

License: (c) (1) This work is licensed under a Creative Commons Attribution 4.0 International License. Read Full License 


\section{Abstract}

Titanium-based nanomaterials co-doped with terbium ( $\mathrm{Tb}$ ) and sulfur $(\mathrm{S})$ were fabricated by sol-gel method via a facile step. Physicochemical properties of the resulting composites were characterized by X-ray diffraction (XRD), Scanning electron microscope (SEM), X-ray photo-electron spectroscopy (XPS) and UV-vis diffuse reflectance spectroscopy (DRS). Methylene blue (MB) was used as a degradation target for evaluating the photocatalytic performance. The factors which influence the photocatalytic activity were investigated, including calcined temperatures and S doping amount. $\mathrm{Tb}, \mathrm{S}(2 \mathrm{wt} \%)$ co-doped $\mathrm{TiO}_{2}$ composite calcined at $600^{\circ} \mathrm{C}$ exhibited the highest photocatalytic activity with a degradation rate of $72.4 \%$ in $3 \mathrm{~h}$. The reaction constant was $0.11529,0.26025,0.35038$, and $0.41462 \mathrm{~h}^{-1}$ for undoped $\mathrm{TiO}_{2}$, Tb-doped $\mathrm{TiO}_{2}$, S-doped $\mathrm{TiO}_{2}$, and $\mathrm{Tb}, \mathrm{S}$ co-doped $\mathrm{TiO}_{2}$, respectively. Importantly, synergistic effect of terbium and sulfur dopants was profoundly discussed. Furthermore, recycling tests and acute toxicity experiments were carried out to confirm the reusability and biosafety of $\mathrm{Tb}, \mathrm{S}$ co-doped $\mathrm{TiO}_{2}$.

\section{Introduction}

Heterogeneous photocatalysis is considered to be one of the most promising technologies in wastewater treatment, particularly for dye contaminants (Chiu et al. 2019). Most of these contaminants are carcinogenic, harmful and can't be degraded by biological methods due to their complex structure and excellent stability, which destroys the aquatic ecosystem and threatens human health (Nguyen et al. 2018; Zhao et al. 2020). In general, catalysts are of significant importance in a photocatalytic process. Among various oxide semiconductor phtocatalysts, $\mathrm{TiO}_{2}$ has attracted much attention as an ideal photocatalytic material due to its strong oxidation ability, cost effectiveness, environmental friendliness, long term stability against photo and chemical corrosion (Kumaravel et al. 2019; Yan et al. 2019; Ananpattarachai et al. 2016). However, its practical applications are restricted by some shortcomings, among which the most serious one is the wide band gap $(3.2 \mathrm{eV})$, resulting in low utilization of sunlight (Karthikeyan et al. 2020). In addition, $\mathrm{TiO}_{2}$ exhibits relatively high electronhole recombination rate which is unfavorable for improving the photocatalytic performance (Tbessi et al. 2019). Therefore, modified materials are in demand for development to overcome the inherent weaknesses of $\mathrm{TiO}_{2}$.

Generally, the photocatalytic activity of $\mathrm{TiO}_{2}$ depends on multiple factors that affect the separation efficiency of electron-hole pairs, such as crystal size, phase, and surface area (Rahmanian et al. 2018). Considerable attempts have been made to the modification of traditional $\mathrm{TiO}_{2}$, consisting of ion doping, dye sensitization, precious metal deposition and semiconductor coupling (Zhou et al. 2016; Wang et al. 2018; Gao et al. 2017; Nzaba et al. 2018). Notably, ion doping, including metal and non-metallic ions, has been regarded as a facile and effective modification method (Wang et al. 2018; Malathi et al. 2018; Ren et al. 2017). However, the recombination centers of the impurity band partially occupied which caused by mono-doping may inhibit the transfer of charge carriers to the surface of $\mathrm{TiO}_{2}$, leading to a reduction of photo-generated current, thus the titanium dioxide couldn't be fully utilized (Yao et al. 2017). Accordingly, double-doped and multi-doped photocatalysts have aroused general interest.

In particular, $\mathrm{TiO}_{2}$ co-doped with rare earth ions and non-metallic elements has greatly attracted people's attention. Rare earth ions have a special $4 \mathrm{fx} 5 \mathrm{dy}$ electronic configuration, which could react with various Lewis bases including acids, alcohols, amines, aldehydes and thiols to forming the complexes (Nasir et al. 2016). It is favorable for the transfer of interfacial charges to the contaminants and leads to the formation of new energy levels between valence band, conduction band and oxygen vacancies. Moreover, rare earth ions could substitute for $\mathrm{Ti}^{4+}$ and significantly reduce the recombination rate of electron-hole pairs (Zhou et al. 2017). In our previous work, it has been proved that terbium doping is an effective means to improve photocatalytic activity, based on its special electronic configuration, variable valence and the ability to generate more energy levels (Wang et al. 2019). For non-metallic ions doping, sulphur has been confirmed that could provide obvious beneficial effects for photocatalytic reactions. It's conducive to reduce the band gap and increase the quantum yield through mixing $p$ states of $S$ with $02 p$ states in titanium dioxide. Besides, surface modification is of great significance in raising adsorption of contaminants and facilitating desorption of oxidation products (Qian et al. 2019). Although the contribution of terbium and sulfur for improving the photocatalytic performance of traditional titanium dioxide is well known, to our best knowledge, Tb, S co-doping in titanium dioxide hasn't been reported, especially the synergistic effect of dopants and its specific photocatalytic mechanism (Spanu et al. 2017). Therefore, combining the advantages of these both dopants and conducting in-depth research on their related theories would be a very meaningful attempt.

In this work, we fabricated Tb, $\mathrm{S}$ co-doped $\mathrm{TiO}_{2}$ nanocomposites by a facile and feasible route. Methylene blue (MB) was applied to evaluate the photocatalytic performance. The factors which influence the photocatalytic activity were investigated in detail. Besides, recycling tests and acute toxicity experiments were conducted to confirm the reusability and biosafety of $\mathrm{Tb}, \mathrm{S}$ co-doped $\mathrm{TiO}_{2}$ composites, respectively. Importantly, synergistic effect of Tb and S co-dopants was further discussed. 


\section{Experimental}

\subsection{Materials}

Methylene blue ( $\geq 99.7 \%$ purity) was supplied by the company of Shanghai macklin biochemical technology. Tetrabuty titanate is of analytical grade and can be used without further purification. Tetrabuty titanate, terbium (III) nitrate hexahydrate and thioureal were purchased from Sigma-Aldrich Chemical Company. Zebrafish $(2.60 \pm 0.45 \mathrm{~cm}$ in length) used in experiments were obtained from aquarium. The fish was placed under natural light and domesticated at room temperature $\left(25 \pm 1^{\circ} \mathrm{C}\right)$ for seven days before the acute toxicity experiments. Dissolved oxygen of water was in the range of 7.21-8.14 $\mathrm{mg} \cdot \mathrm{L}^{-1}$. Note that the aeration was performed continuously.

\subsection{Characterization}

Crystal structure of the photocatalysts was measured by a D8 Advance X-ray diffractometry (Bruker, Germany). The test was operated from $10^{\circ}$ to $80^{\circ}(2 \theta)$, and Cu Ka was used as the radiation source. Crystalline sizes (D) of the resulting photocatalysts was calculated by Scherrer's equation $D=K \lambda / B \cos \theta$. XPS spectra were recorded on an AXIS SUPRA spectroscopy (Shimadzu, Japan) to analyze the surface elements and chemical valence. UV-Vis diffuse reflectance spectra were performed by a Lambda 750s spectrophotometer (Perkinelmer, USA), using white barium sulfate as the reference. Morphology image was obtained from a S-4800 scanning electron microscope (Hitachi, Japan). PL spectra were measured by a F-4600 spectrophotometer with a excitation wavelength of $315 \mathrm{~nm}$ (Hitachi, Japan). U-2910 UV-Vis spectrophotometer (Hitachi, Japan) was used to measure the absorbance of MB.

\subsection{Photocatalysts preparation}

Tb, S co-doped $\mathrm{TiO}_{2}$ nanocomposites were fabricated by sol-gel method via a facile step. In a typical preparation, $20 \mathrm{~mL}$ of TBT and $25 \mathrm{~mL}$ of absolute ethanol were mixed under vigorous stirring at room temperature, which namely solution A. Meanwhile, $0.1335 \mathrm{~g}$ of terbium(III) nitrate hexahydrate, $20 \mathrm{~mL}$ of deionized water, $10 \mathrm{~mL}$ of glacial acetic acid, $25 \mathrm{~mL}$ of absolute ethanol and an appropriate amount of thiourea were added to a beaker, the stirring was continued for $0.5 \mathrm{~h}$ to form solution B. Thereafter, solution B was slowly added to solution $\mathrm{A}$, and the reaction temperature was adjusted to $70^{\circ} \mathrm{C}$. The uniform $\mathrm{TiO}_{2}$ sol was obtained after $2 \mathrm{~h}$, and subsequently subjected to aging and drying procedure. Finally, the resulting photocatalysts were obtained after the mixture calcined in a muffle furnace for $4 \mathrm{~h}$ under different temperatures $\left(300^{\circ} \mathrm{C}, 400^{\circ} \mathrm{C}, 500^{\circ} \mathrm{C}, 600^{\circ} \mathrm{C}\right)$. It is worth mentioning that the $\mathrm{Tb}$ amount was kept constant, and S-doping ratio was set to $1 \%$, $2 \%, 3 \%$ and $4 \%$, respectively (Wang et al. 2019). For comparison, pure $\mathrm{TiO}_{2}$, Tb- and S- mono-doped $\mathrm{TiO}_{2}$ nanomaterials were also fabricated via similar step.

\subsection{Photocatalytic evaluation}

The photocatalytic activity of the catalysts was evaluated by degradation of $10 \mathrm{mg} \cdot \mathrm{L}^{-1} \mathrm{MB}$ in aqueous solution with $0.1 \mathrm{~g}$ of the catalysts. Prior to photocatalytic reaction, dark experiments $(0.5 \mathrm{~h})$ were carried out for the sufficient adsorption of dye molecules on the catalysts. In a typical photocatalytic process, continuous magnetically stirring was kept under light irradiation. At specific time intervals, $5 \mathrm{ml}$ of the suspension was taken and centrifuged. Changes in MB concentration was measured via the absorbance values at $662 \mathrm{~nm}$.

\subsection{Acute toxicity experiments}

Acute toxicity experiments were conducted with a static method. In other words, keeping the zebrafishes without eating and the test solutions were not replaced during the experiments. Firstly, $5 \mathrm{~L}$ of water and 15 zebrafishes were put into each aquarium. After that, adding an appropriate amount of $\mathrm{Tb}, \mathrm{S}$ co-doped $\mathrm{TiO}_{2}$ composites to control solution concentrations to be $0,12.5 \mathrm{mg} \cdot \mathrm{L}^{-1}, 25 \mathrm{mg} \cdot \mathrm{L}^{-1} \mathrm{and} 50 \mathrm{mg} \cdot \mathrm{L}^{-1}$, respectively. Recorded the survival of zebrafishes at a specific time, and promptly removed dead zebrafishes. Finally, the mortality were calculated at $24,48,72$, and $96 \mathrm{~h}$, respectively, and the half lethal concentration $\left(\mathrm{LC}_{50}\right)$ was further determined.

\section{Results And Discussion}

\subsection{Photocatalysts Characterization}

Generally, the anatase has more oxygen vacancies than rutile, it is easier to capture electrons and effectively suppress the recombination of electron-hole pairs, thus the photocatalytic activity of anatase is higher than that of rutile (Spanu et al. 2017). Therefore, the calcined temperatures were controlled in the range of $300-600^{\circ} \mathrm{C}$ to obtain the samples with good photocatalytic performance. XRD patterns of Tb, $S$ co-doped $\mathrm{TiO}_{2}$ composites under different calcined temperatures are presented in Fig. 1 (a). Peaks at $2 \theta$ of $25.38^{\circ}, 37.88^{\circ}, 48.10^{\circ}, 54.10^{\circ}$, $55.28^{\circ}$, and $62.75^{\circ}$ would be the characteristics for (101), (004), (200), (105), (211), and (204) of anatase, respectively (PDF N0.65-5714), indicating that no rutile phase diffraction was detected for all of the composites under different calcined temperatures. On the basis of XRD 
patterns, the crystallite size of different samples was calculated by Scherrer's equation, as listed in Table 1 . The increasing of calcined temperatures resulted in an enhancement of the crystallite size. $\mathrm{Tb}, \mathrm{S}$ co-doped $\mathrm{TiO}_{2}$ composite calcined at $500^{\circ} \mathrm{C}$ was with a crystallite size of $11.5 \mathrm{~nm}$, less than that of undoped $\mathrm{TiO}_{2}$ calcined at $500^{\circ} \mathrm{C}(12.7 \mathrm{~nm})$. It suggested that Tb and $\mathrm{S}$ co-doping could inhibit grain growth of $\mathrm{TiO}_{2}$, owing to the deformation of lattice and oxygen vacancies left by the substitution of $\mathrm{O}$ atoms for $\mathrm{Tb}$ or $\mathrm{S}$ atoms. Correspondingly, specific surface area of $\mathrm{Tb}, \mathrm{S}$ co-doped $\mathrm{TiO}_{2}$ composite calcined at $500^{\circ} \mathrm{C}$ was larger than that of undoped $\mathrm{TiO}_{2}$. Fig. $1(\mathrm{~b})$ shows the $\mathrm{SEM}$ images of $\mathrm{Tb}, \mathrm{S}$ co-doped $\mathrm{TiO}_{2}$ composites calcined at $500^{\circ} \mathrm{C}$. Regularly shaped agglomerate spherical particles with diameters of about 17 $\mathrm{nm}$ were clearly observed.

Table 1

Physical properties of different samples.

\begin{tabular}{|c|c|c|c|}
\hline Samples & Crystallinity (\%) a & Crystallite size $(\mathrm{nm})^{\mathrm{b}}$ & $\operatorname{BET}\left(\mathrm{m}^{2} / \mathrm{g}\right)$ \\
\hline $\mathrm{Tb}, \mathrm{S}$ co-doped $\mathrm{TiO}_{2}-300^{\circ} \mathrm{C}$ & 93.44 & 9.9 & 94 \\
\hline $\mathrm{Tb}, \mathrm{S}$ co-doped $\mathrm{TiO}_{2}-400^{\circ} \mathrm{C}$ & 95.07 & 10.8 & 89 \\
\hline $\mathrm{Tb}, \mathrm{S}$ co-doped $\mathrm{TiO}_{2}-500^{\circ} \mathrm{C}$ & 98.97 & 11.5 & 82 \\
\hline $\mathrm{Tb}, \mathrm{S}$ co-doped $\mathrm{TiO}_{2}-600^{\circ} \mathrm{C}$ & 99.56 & 13.9 & 73 \\
\hline Undoped $\mathrm{TiO}_{2}-500^{\circ} \mathrm{C}$ & 99.68 & 12.7 & 76 \\
\hline \multicolumn{4}{|c|}{ a Evaluated by Jade 5.0 software. } \\
\hline
\end{tabular}

XPS analysis was performed to analyze the surface elements and chemical valence. Fig. 2 presents the $01 s$, Ti $2 p$, Tb $3 d$, and $S 2 p$ spectra of the $\mathrm{Tb}, \mathrm{S}$ co-doped $\mathrm{TiO}_{2}$ composite. Two peaks located at 528.63 and $530.3 \mathrm{eV}$ could be observed in 0 1s spectra (Fig. $2 \mathrm{a}$ ), which were ascribed to Ti-O and superficial oxygen of chemically adsorbed hydroxyl species (Liu et al. 2019). The results of the XPS spectra for Ti $2 p$ are displayed in Fig. 2(b). It could be fitted into four peaks, which were assigned to the characteristic peaks of $\mathrm{Ti}^{3+}$ and $\mathrm{Ti}^{4+}$ state $(\mathrm{Wang}$ et al. 2018). Note that the presence of $\mathrm{Ti}^{3+}$ might be caused by the reduction of $\mathrm{Ti}^{4+}$ in the preparation process. This interesting phenomenon could induce defects in the $\mathrm{TiO}_{2}$ lattice, and further resulted in the formation of oxygen vacancies. As seen from Fig. 2(c), two broad spinorbit doublet peaks at 1226.84 and $1242.7 \mathrm{eV}$ belong to $\mathrm{Tb}^{3+} 2 \mathrm{p}_{5 / 2}$ and $\mathrm{Tb}^{3+} 2 \mathrm{p}_{3 / 2}$, respectively, which was indicative of the formation of $\mathrm{Tb}_{2} \mathrm{O}_{3}$ and shallow traps on the surface of photocatalysts (Wang et al. 2019). Accordingly, the carriers could be quickly captured, facilitating the separation of photogenerated electrons and holes. For S $2 p$ spectra (Fig. $2 \mathrm{~d}$ ), the peaks at $168.0 \mathrm{eV}$ and $167.3 \mathrm{eV}$ were ascribed to $\mathrm{S}^{4+}$ $2 p_{3 / 2}$ and $S^{4+} 2 p_{1 / 2}$. Generally, the substitution of $\mathrm{Ti}^{4+}$ by cationic $S$ is energetically more favorable than replacing $\mathrm{O}^{2-}$ with $\mathrm{S}^{2-}(\mathrm{Vorontsov}$ et al. 2019). It could result in a charge imbalance in the lattice, and the excess positive charge might be neutralized by hydroxide ions, thus the recombination of electrons and holes was effectively suppressed. Besides, the binding energy related to $S^{6+} 2 p_{1 / 2}$ and $S^{6+} 2 p_{3 / 2}$ levels has confirmed the formation of $\mathrm{SO}_{4}{ }^{2-}$ on the surface of $\mathrm{TiO}_{2}$, which could effectively capture the photo-generated electrons and holes. All of these results demonstrated that $\mathrm{Tb}, \mathrm{S}$ co-doping was favorable for the improvement of photocatalytic performance.

\subsection{Factors influencing the photocatalytic efficiency}

Calcined temperature and doping amount of the elements are two important factors affecting the photocatalytic performance. It has been reported that calcined temperature profoundly influenced the structural characteristics, including crystallinity, crystallite size and specific surface. Owing to the change in structural characteristics, the surface defects and active sites of the photocatalyst were further changed (Liu

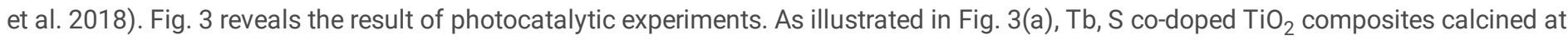
$500^{\circ} \mathrm{C}$ exhibited the highest photocatalytic performance with a degradation rate of $72.4 \%$ in $3 \mathrm{~h}$. The reduced performance of photocatalyst with high calcined temperature should be due to the destruction of the structured morphology and decrease of the specific surface area (Table 1). On the contrary, low-temperature calcined catalysts with weak purification, more defects and low active sites also led to poor photocatalytic performance. Therefore, $500^{\circ} \mathrm{C}$ was chosen as the optimal calcined temperature for further studies. In addition to calcined temperature, the effect of S doping amount on the photocatalytic performance was also investigated. As shown in Fig. 3(b), the increment of $S$ doping amount from 1-2\% resulted in an enhancement of the photocatalytic activity. However, a decrease of the degradation rate was obviously observed in the range of $2 \%-4 \%$. The decrease in photocatalytic activity was attributed to the presence of excess sulfur ions, which acted as recombination centers for electrons and holes pairs (Gyorgy et al. 2018). Moreover, it could absorb incident photons and reduce the 
light exposed to the catalyst, subsequently the photocatalytic activity decreased. Therefore, $2 \%$ was chosen as the optimal doping amount for further studies.

\subsection{Synergistic effect discussion}

In order to investigate the synergistic effect of co-dopants in a photocatalytic process, $\mathrm{TiO}_{2}, \mathrm{~S}-, \mathrm{Tb}^{-}$and $\mathrm{Tb}, \mathrm{S}$ co-doped $\mathrm{TiO}_{2}$ were used as catalysts for comparative experiments. The photocatalytic performance of MB degraded by different catalysts is displayed in Fig. 4(a). It worth emphasizing that the photocatalytic performance of doped catalysts was better than undoped catalysts. Notably, $\mathrm{Tb} \mathrm{S}$ co-doped $\mathrm{TiO} \mathrm{O}_{2}$ composite presented a remarkable increment in the photocatalytic performance with a degradation rate of 72.4\%. As revealed in Fig. 4(b), good linear relationship between $-\ln \left(C_{t} / C_{0}\right)$ and irradiation time were observed in all cases, indicating that MB degradation was followed a pseudo-first-order pattern. Reaction rate constant(k) was determined by the slope of graph plotted between $-\ln \left(C_{t} / C_{0}\right)$ and irradiation time. The value of $\mathrm{k}$ was $0.11529,0.26025,0.35038$, and $0.41462 \mathrm{~h}^{-1}$ for $\mathrm{TiO}_{2}, \mathrm{~Tb}$-, S- and $\mathrm{Tb}, \mathrm{S}$ co-doped $\mathrm{TiO}_{2}$, respectively. The reaction rate of $\mathrm{Tb}, \mathrm{S}$ co-doped $\mathrm{TiO}_{2}$ composites was approximately 3.6 times compared with pure $\mathrm{TiO}_{2}$, which might be attributed to the synergistic effect of terbium and sulfur dopants. The UV-vis diffraction reflectance spectra of photocatalysts are illustrated in Fig. $4(\mathrm{c})$. $\mathrm{Pure} \mathrm{TiO}_{2}$ was chosen as a reference. It is clearly observed that the absorption edge of S-doped and Tb, S co-doped samples exhibited an slight red-shift, whereas Tb-doped sample showed no obvious change into the visible region. The order of the redshift for different samples is listed as follows: Tb-S$\mathrm{TiO}_{2} \approx \mathrm{S}-\mathrm{TiO}_{2}>\mathrm{Tb}-\mathrm{TiO}_{2} \approx \mathrm{TiO}_{2}$, which arose from the contribution of S dopant. In addition, doping of mono- or double- ions would cause difference between light absorption capacity in ultraviolet region. The intensity of the absorption peaks can be arranged in the following order, $\mathrm{Tb}-\mathrm{S}-\mathrm{TiO}_{2}>\mathrm{S}-\mathrm{TiO}_{2}>\mathrm{Tb}-\mathrm{TiO}_{2}>\mathrm{TiO}_{2}$, suggesting that $\mathrm{Tb}$ and $\mathrm{S}$ dopants have an obvious synergistic effect on improving the photocatalytic efficiency. Fig. 4(d) presents the UV-vis spectra of MB in a degradation process. The absorbance was decreased with the enhancement of irradiation time, which was indicative of the gradually degradation of $\mathrm{MB}$ by $\mathrm{Tb}, \mathrm{S}$ co-doped $\mathrm{TiO}_{2}$ composites. Taking advantage of the synergistic effect of terbium and sulfur dopants, $\mathrm{Tb}, \mathrm{S}$ co-doped $\mathrm{TiO}_{2}$ composites exhibited obvious enhancement in the photocatalytic activity. Tb, S co-doping could strengthen the response to light irradiation and facilitate the separation of electron-holes. On the other hand, $\mathrm{Tb}, \mathrm{S}$ co-doped $\mathrm{TiO}_{2}$ composites with smaller average crystallite size could provide larger specific surface area and more active centers, which was favorable for the adsorption between dye molecules and catalysts. Besides, the smaller crystallite size was beneficial for the rapid transfer of photogenerated electrons and holes to the surface of the $\mathrm{TiO}_{2}$ (Gyorgy et al. 2018).

Formation process of $\cdot \mathrm{OH}$ was confirmed by a $\mathrm{PL}$ spectroscopy. Terephthalic acid was applied as a scavenger, and the experimental details were the same as previously reported (Wang et al. 2019; Amer al. 2019). Fluorescence spectra of $\bullet \mathrm{OH}$ tracking process is illustrated in Fig. 4(e). A positive relationship between the photocatalytic degradation and the amount of $\cdot \mathrm{OH}$ radicals was observed, which demonstrated that $\cdot \mathrm{OH}$ radicals have been one of the primary reactive species responsible for the degradation of dye contaminants. Electrons( $\left.\mathrm{e}^{-}\right)$and holes $\left(\mathrm{h}^{+}\right)$were generated by photons under light irradiation, and electrons transferred from the valence band (VB) to the conduction band (CB). However, these charge carriers were easily recombined, resulting in low photocatalytic performance (Santacruz et al. 2020; Cheng et al. 2016). After doping of terbium ion, it could be served as trapping centers for the photogenerated electrons to reduce the recombination rate, which allowed the catalyst to produce more active species and improve the photoactivity.

\subsection{Photoatalysts re-usability}

Reusability is one of the major concerns for the practical applications of photoatalysts ${ }^{[28]}$. Therefore, four successive recycling tests for the degradation of MB were conducted to investigate the photocatalyst reusability. In a typical experiment, the catalysts were collected by a centrifugal process after each run. The separated photocatalysts were washed thoroughly with deionized water and ethanol, and dried at $105^{\circ} \mathrm{C}$ for $3 \mathrm{~h}$ before the beginning of next cycle. Note that the photocatalysts should be calcined at $500^{\circ} \mathrm{C}$ before use of the fourth cycle. It can be observed from Fig. 5 that the degradation rate was $72.4 \%, 68.2 \%$ and $63.7 \%$ for the first to the third runs, respectively. Slight decrease in the degradation rate was mainly due to the absorbed intermediates on the surface or interface of the catalyst as well as to the loss of a small amount of photocatalyst during the recovery process. It worth emphasizing that the degradation rate of the fourth cycle was only $2.2 \%$ less than that of the fresh cycle, demonstrating that a calcined process was favorable for the regeneration of the photocatalysts. In conclusion, re-usability tests have confirmed that the resulting $\mathrm{Tb}, \mathrm{S}$ co-doped $\mathrm{TiO}_{2}$ composites owned good stability and could be potentially employable for continuous photocatalytic processes.

\subsection{Acute toxicity of photocatalysts}

Biological safety of photocatalysts is of great significance for their potential applications in wastewater treatment. Herein, we evaluated the effects of $\mathrm{Tb}, \mathrm{S}$ co-doped $\mathrm{TiO}_{2}$ on the aquatic ecosystem via acute toxicity experiments. As presented in Fig. 6, mortality of zebrafish was increased with the increasing of catalyst concentrations. Tb, S co-doped $\mathrm{TiO}_{2}$ showed low toxicity on zebrafishes with a half lethal concentration of $20.3 \mathrm{mg} \cdot \mathrm{L}^{-1}\left(96 \mathrm{~h}-\mathrm{LC}_{50} \otimes 10 \mathrm{mg} \cdot \mathrm{L}^{-1}\right)$. 


\section{Conclusions}

Terbium and sulfur co-doped $\mathrm{TiO}_{2}$ composites were successfully fabricated by sol-gel method via a facile step. All the samples existed in the pattern of only anatase structure. The crystallite size of $\mathrm{Tb}, \mathrm{S}$ co-doped $\mathrm{TiO}_{2}$ calcined at $500^{\circ} \mathrm{C}(11.5 \mathrm{~nm})$ was less than undoped $\mathrm{TiO}_{2}$. According to the XPS results, sulfur was doped in the forms of $\mathrm{S}^{4+}$ and $\mathrm{S}^{6+}$ cations and oxidation state of terbium was $\mathrm{Tb}^{3+}$. The resulting Tb-S (2 wt\%) co-doped $\mathrm{TiO}_{2}$ composite calcined at $500^{\circ} \mathrm{C}$ exhibited the highest photocatalytic performance with a reaction constant of $0.41462 \mathrm{~h}^{-1}$. Owing to the synergistic effect, $\mathrm{Tb}$ and $\mathrm{S}$ dopants in the structure of $\mathrm{TiO}_{2}$ resulted in a remarkable strengthening response to light irradiation and caused significant separation of the electron-hole pairs. Moreover, smaller crystallite of the Tb, S co-doped $\mathrm{TiO}_{2}$ composite could provide more active centers, facilitating the interaction between organic molecules and photocatalysts, thereby effectively improving the photocatalytic activity. The photocatalysts have been proved to be used repeatedly at least four cycles and only a slight decline in photocatalytic activity was observed. Acute toxicity experiments demonstrated that the photocatalysts presented low toxicity on zebrafishes with a half lethal concentration of $20.3 \mathrm{mg} \cdot \mathrm{L}^{-1}$. All these results reveal that the promising prospects of $\mathrm{Tb}, \mathrm{S}$ co-doped $\mathrm{TiO}_{2}$ composite in the practical application of water treatment.

\section{Declarations}

\section{Ethical Approval}

The experimental protocols were approved by the Animal Care and Protection Committee of Hainan Medical University.

\section{Consent to Participate}

All authors agreed to be held accountable for the work performed therein.

\section{Consent to Publish}

All authors gave final approval for publication.

\section{Authors Contributions}

Zhencui Wang participated in the experimental operation and drafted the manuscript; Yuanjiao Wang collected and analyzed the data.Tianle Tang conceived, designed and coordinated the study and helped draft the manuscript.

\section{Funding}

This project was financially supported by Natural Science Foundation of Hainan Province (219QN220) and the Scientific research Foundation of Hainan Medical University (HY2018-17).

\section{Competing Interests}

The authors declare that they have no known competing financial interests or personal relationships that could have appeared to influence the work reported in this paper.

\section{Availability of data and materials}

Not applicable.

\section{References}

1. AmerMS, ArunachalamP, Al-MayoufAM, PrasadS, AlshalwiMN,GhanemMA(2019) Mesoporous tungsten trioxide photoanodes modified withnitrogen-doped carbon quantum dots for enhanced oxygen evolution photo-reaction.Nanomaterials: 9 1502-1507.

2. AnanpattarachaiJ, SeraphinS, KajitvichyanukulP (2016) Formation of hydroxyl radicals and kinetic studyof 2-chlorophenol photocatalytic oxidation using C-doped $\mathrm{TiO}_{2}, \mathrm{~N}$-doped $\mathrm{TiO}_{2}$, and $\mathrm{C}, \mathrm{N}$ Co-doped $\mathrm{TiO}_{2}$ under visible light. Environmental Science Pollution Research, 23: 3884-3896.

3. ChiuYH, ChangTM, ChenCY,SoneM, HsuYJ (2019) Mechanistic insightsinto photodegradationof organic dyes using heterostructure photocatalysts. Catalysts, 9: 430-462. 
4. ChengZ, GuZ, ChenJ, YuJ, ZhouL (2016) Synthesis, characterization, and photocatalytic activity ofporous La-N-co-doped TiO 2 nanotubes for gaseouschlorobenzene oxidation. Journal of Environmental Sciences, 46:203-213.

5. GaoH, LiuJ, ZhangJ, ZhuZ, ZhangG, LiuQ (2017) Influence of carbon and yttrium co-doping on the photocatalyticactivity of mixed phase $\mathrm{TiO}_{2}$. Chinese Journal of Catalysis, 38:1688-1696.

6. GyorgyE, LogofatuC, PinoAP, DatcuA, PascuO, IvanR(2018) Enhanced UV-and visible-light driven photocatalytic performances and recycling properties of graphene oxide/ZnO hybrid layers. Ceramics International, 44:1826-1835.

7. Kumaravel V, Mathew S, Bartlett J, Pilla S (2019)Photocatalytichydrogenproductionusingmetaldoped $\mathrm{TiO}_{2}:$ Areviewofrecentadvances.Applied Catalysis B: Environmental,244: 1021-1064.

8. Karthikeyan C, Arunachalam P, Ramachandran K, Mayouf A, Karuppuchamy S (2020) Recentadvancesinsemiconductormetaloxideswithenhancedmethodsforsolarphotocatalyticapplications. JournalofAlloysandCompounds, 828: 154281-154295.

9. KaurR, VellingiriK, KimKH, PaulAK, DeepA (2016) Efficient photocatalytic degradation of rhodamine 6Gwith a quantum dot-metal organic framework nanocomposite. Chemosphere, 154:620-627.

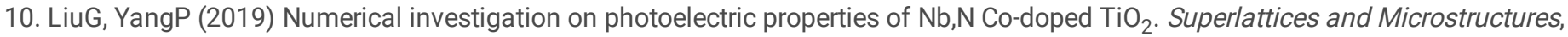
129:130-138.

11. LiuJ, KeJ, LiY, LiuB, WangL, XiaoH, WangS(2018) $\mathrm{Co}_{3} \mathrm{O}_{4}$ quantum dots/ $\mathrm{TiO}_{2}$ nanobelt hybrids for highly efficient photocatalytic overall water splitting. Applied Catalysis B: Environmental, 236:396-403.

12. MalathiA, Madhavan J, AshokkumarM, ArunachalamP(2018)A review on $\mathrm{BiVO}_{4}$

13. photocatalyst: activity enhancement methods for solar photocatalytic applications. Applied Catalysis A, 555:47-74.

14. NzabaS K, NtsendwanaB, MambaB B, KuvaregaA T(2018) PAMAM templated N,Pt co-doped TiO $_{2}$ for visible lightphotodegradation of brilliant black. Environmental Science and Pollution Research, 23:3884-3896.

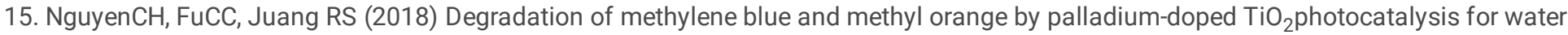
reuse: Efficiency and degradation pathways. Journal of Cleaner Production, 08:110-118.

16. NasirM, LeiJ, IqbalW, ZhangJ (2016) Study of synergistic effect of Sc and C co-doping on the enhancementofvisible light photocatalytic activity of $\mathrm{TiO}_{2}$. Applied Surface Science, 364:446-454.

17. QianY, DuJ, KangD J (2019) Enhanced electrochemical performance of porous co-doped $\mathrm{TiO}_{2}$ nanomaterials prepared by a solvothermal method. Microporous and Mesoporous Materials, 273:148-155.

18. RahmanianE, MalekfarR, PumeraM(2018) Nanohybrids of two-dimensional transition-metal dichalcogenides and titanium dioxide for photocatalytic applications. Chemistry- A European Journal, 24:18-31.

19. RenH, KoshyP, ChenWF, QiS, SorrellCC(2017) Photocatalytic materials andtechnologies for air purification.Journal of Hazardous Materials, 325:340-366.

20. SpanuD, RecchiaS, MohajerniaS, SchmukiP, AltomareM(2018) Site-selective Ptdewetting on $\mathrm{WO}_{3}-\mathrm{Coated}^{\mathrm{TiO}_{2}}$ nanotube arrays: an Electron transfer cascade-based $\mathrm{H}_{2}$ evolution photocatalyst.Applied Catalysis B: Environmental, 237:198-205.

21. SantacruzM R, MunozP G, MarchalC, BatailN, PhamC, RobertD,KellerN(2020) Coating-free TiO ${ }_{2} @ \beta$-SiC alveolar foams as a ready-to-use composite photocatalyst with tunable adsorption properties for water treatment. RSC Advances, 10:3817-3825.

22. Tbessil, BenitoM, MolinsE, LiorcaJ, TouatiA, SayadiS,NajjarW (2019) Effect of Ce and Mn co-doping on photocatalytic performance of sol-gel $\mathrm{TiO}_{2}$. Solid State Sciences, 88 20-26.

23. Vorontsov A, Valdes H (2019) InsightsintothevisiblelightphotocatalyticactivityofS-dopedhydratedTiO 2 . International Journal Hydrogen Energy, 44囚17963-17973.

24. WangS, BaiL M, AoX L (2018) Preparation and photocatalytic application of a S,Nd double doped nano-TiO 2 photocatalyst. $R S C$ Advances, 8:36745-36753.

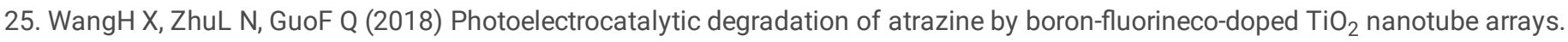
Environmental Science and Pollution Research, 6:11356-11365.

26. WangZ, SongY, CaiX,Zhang J, WenS (2019) Rapid preparation of terbium doped titanium dioxide nanoparticles and its enhanced photocatalytic Performance. Royal Society Open Science, 6:191077-191083.

27. Wang P, ZhouQ, XiaY, ZhanS, LiY(2018)Understanding the charge separation andtransfer in mesoporous carbonate-doped phasejunction $\mathrm{TiO}_{2}$ nanotubes forphotocatalytic hydrogen production.Applied Catalysis B: Environmental, 225: $433-444$.

Page $7 / 10$ 
28. Yan J C, Zhao J, Hao L, Hu Y F, Liu T Y, Guan S J, Zhao Q, Zhu Z, Lu Y (2019) Low-temperatureS-dopingonNdopedTiO ${ }_{2}$ filmsandremarkableenhancementonvisible-lightperformance. Materials Research Bulletin,120: 110594-110600.

29. YaoS, WangS, SunS,Shi Z (2017) Preparation, characterization of Fe, Ce co-doped flower-like $\mathrm{TiO}_{2}$ photocatalysts and their properties in photocatalytic oxidation of arsenite. Russian Journal of Physical Chemistry A, 91:1132-1137.

30. ZhaoD, CaiC (2020) Adsorption and photocatalytic degradation of pollutants on Ce-doped MIL-101- $\mathrm{NH}_{2} / \mathrm{Ag}_{3} \mathrm{PO} \mathrm{O}_{4}$ composites. Catalysis Communications, $136: 105910-105$.

31. ZhouX, ZhangX N, FengX B, ZhouJ, ZhouS Q (2016) Preparation of a La/N co-doped $\mathrm{TiO}_{2}$ film electrode with visible lightresponse and its photoelectrocatalytic activity on a Ni substrate. Dyes and Pigments, 125:375-383.

32. ZhouL, ZhangW, ChenL, DengH, WanJ(2017) A novel ternary visible-light-driven

33. photocatalyst $\mathrm{AgCl} / \mathrm{Ag}_{3} \mathrm{PO}_{4} / \mathrm{g}-\mathrm{C}_{3} \mathrm{~N}_{4}$ : synthesis, characterization, photocatalyticactivity for antibiotic degradation and mechanism analysis. Catalysis Communications, 100:191-195.

\section{Figures}

\section{Figure 1}

(a) XRD patterns of $\mathrm{Tb}, \mathrm{S}\left(2 \mathrm{wt} \%\right.$ ) co-doped $\mathrm{TiO}_{2}$ composites under different calcined temperatures; (b) SEM image of $\mathrm{Tb}, \mathrm{S}$ co-doped $\mathrm{TiO}{ }_{2}$ composites calcined at $500^{\circ} \mathrm{C}$.
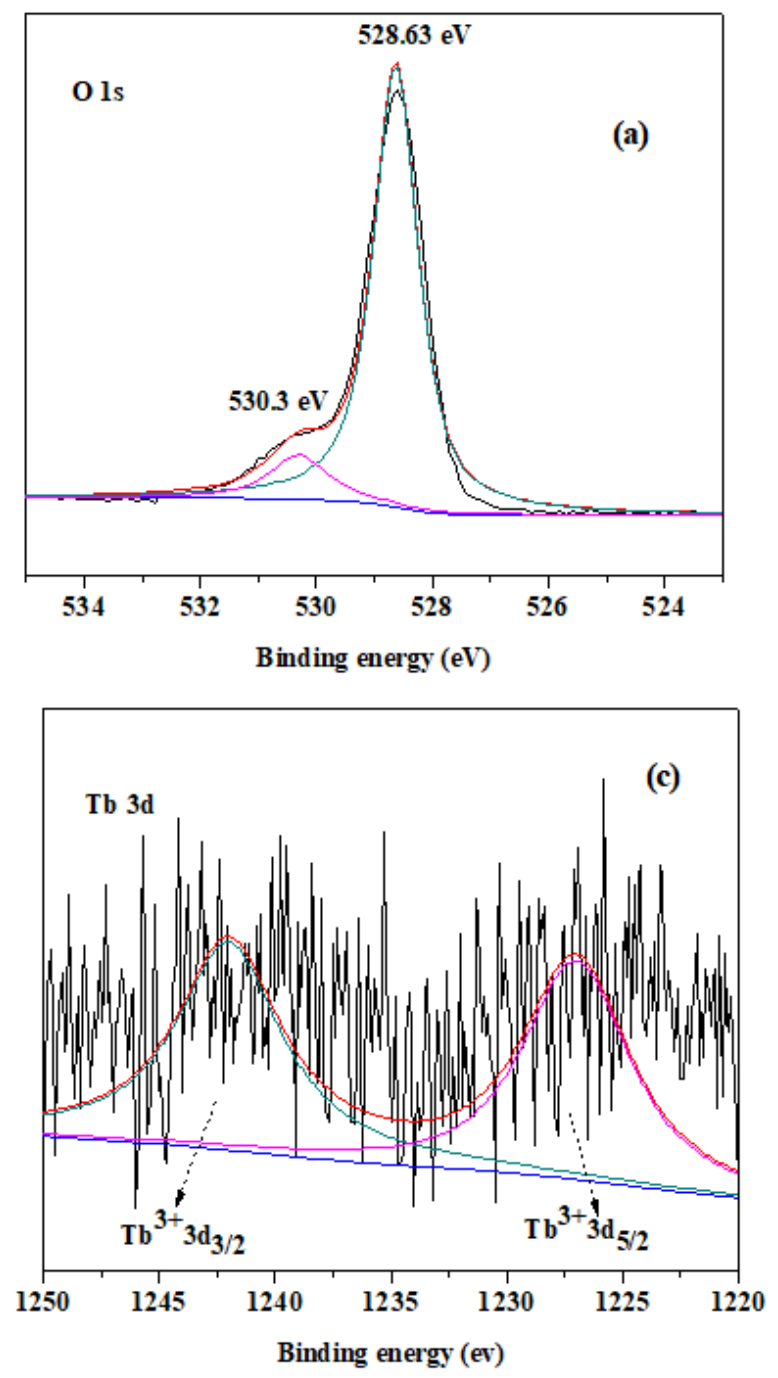
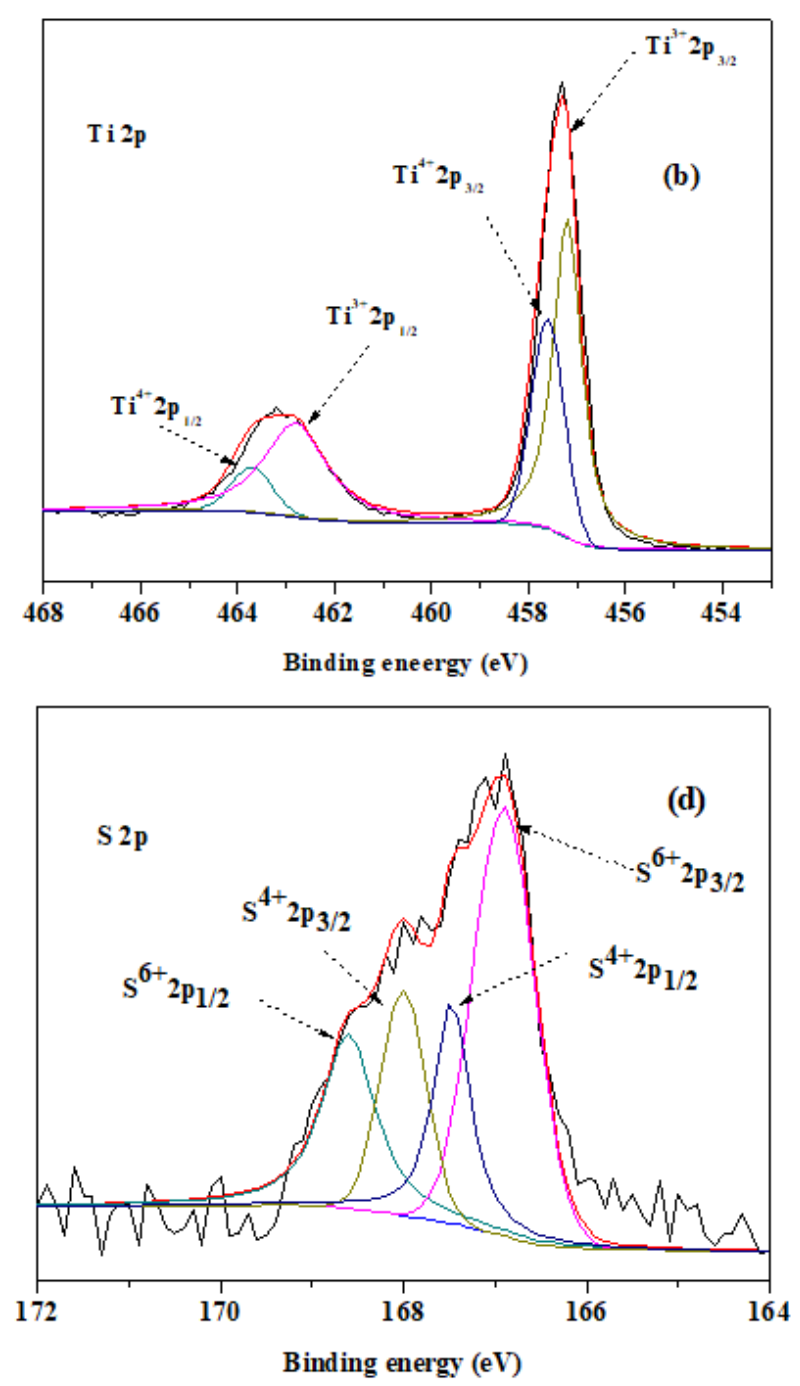

Page $8 / 10$ 
Figure 2

XPS spectra of Tb, S co-doped $\mathrm{TiO}_{2}$ composites:(a)O 1s, (b)Ti 2p, (c)Tb 3d, (d)S 2p.
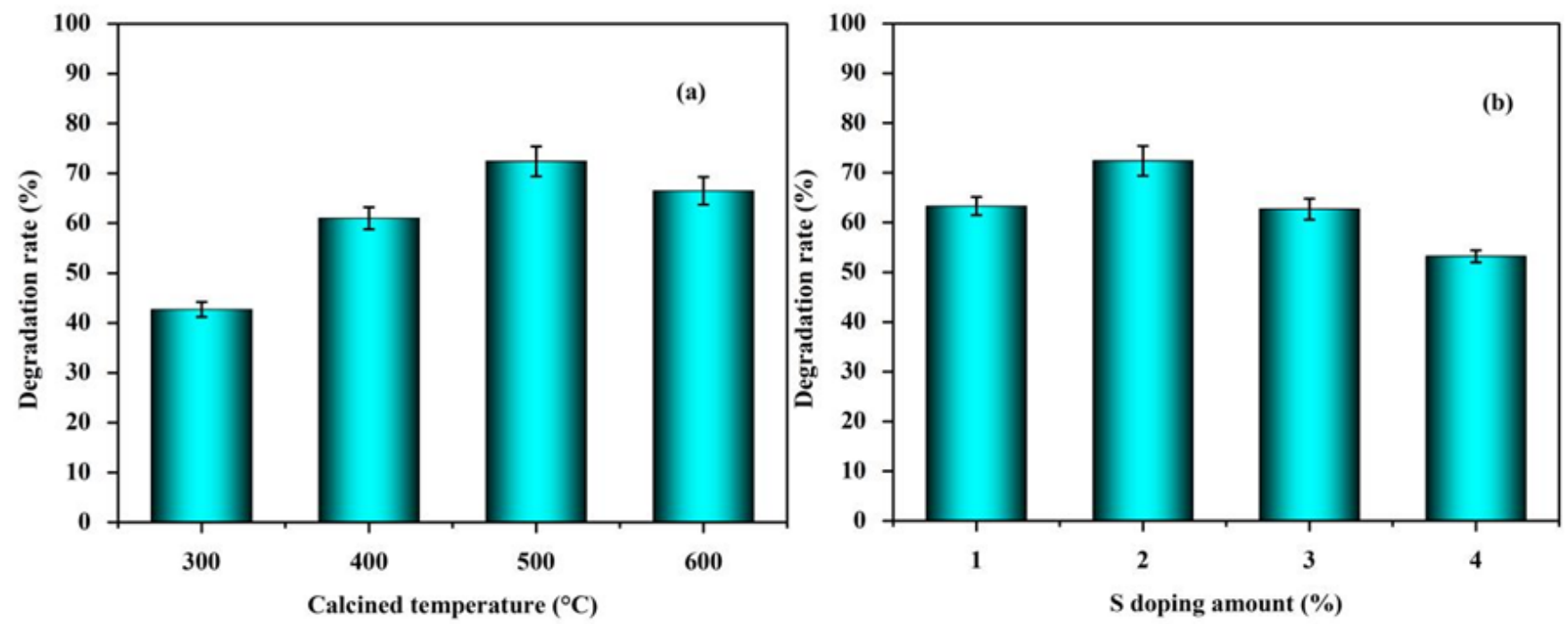

Figure 3

Photocatalytic activity under different conditions:(a)calcined temperatures and (b)S doping amount.
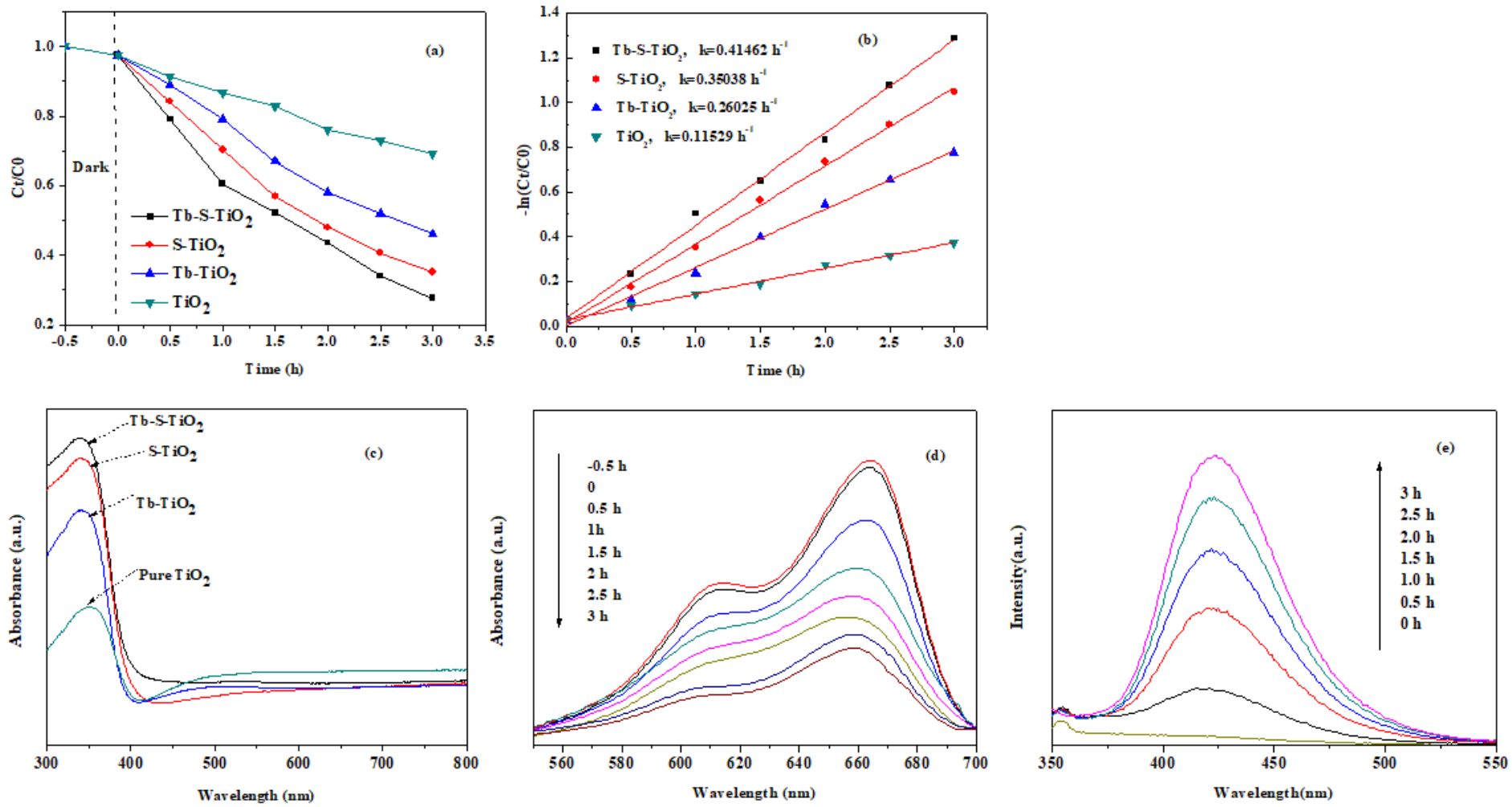

Figure 4

(a)Photocatalytic activity comparison of co-doped and mono-doped titanium dioxide;(b) Plots of $-\ln \left(\mathrm{C}_{\mathrm{t}} / \mathrm{C}_{0}\right)$ versus irradiation time for the degradation of MB over different photocatalysts; (c)UV-Vis diffuse reflectance spectra of pure $\mathrm{TiO}_{2}, \mathrm{~S}$-, Tb-and Tb,S co-doped $\mathrm{TiO}_{2}$; (d) UV-Vis absorption spectra of MBdegraded with Tb, $\mathrm{S}$ co-doped $\mathrm{TiO}_{2}$; (e)Fluorescence spectra of $\cdot \mathrm{OH}$ tracking process of with $\mathrm{Tb}, \mathrm{S}$ co-doped $\mathrm{TiO}_{2}$. 


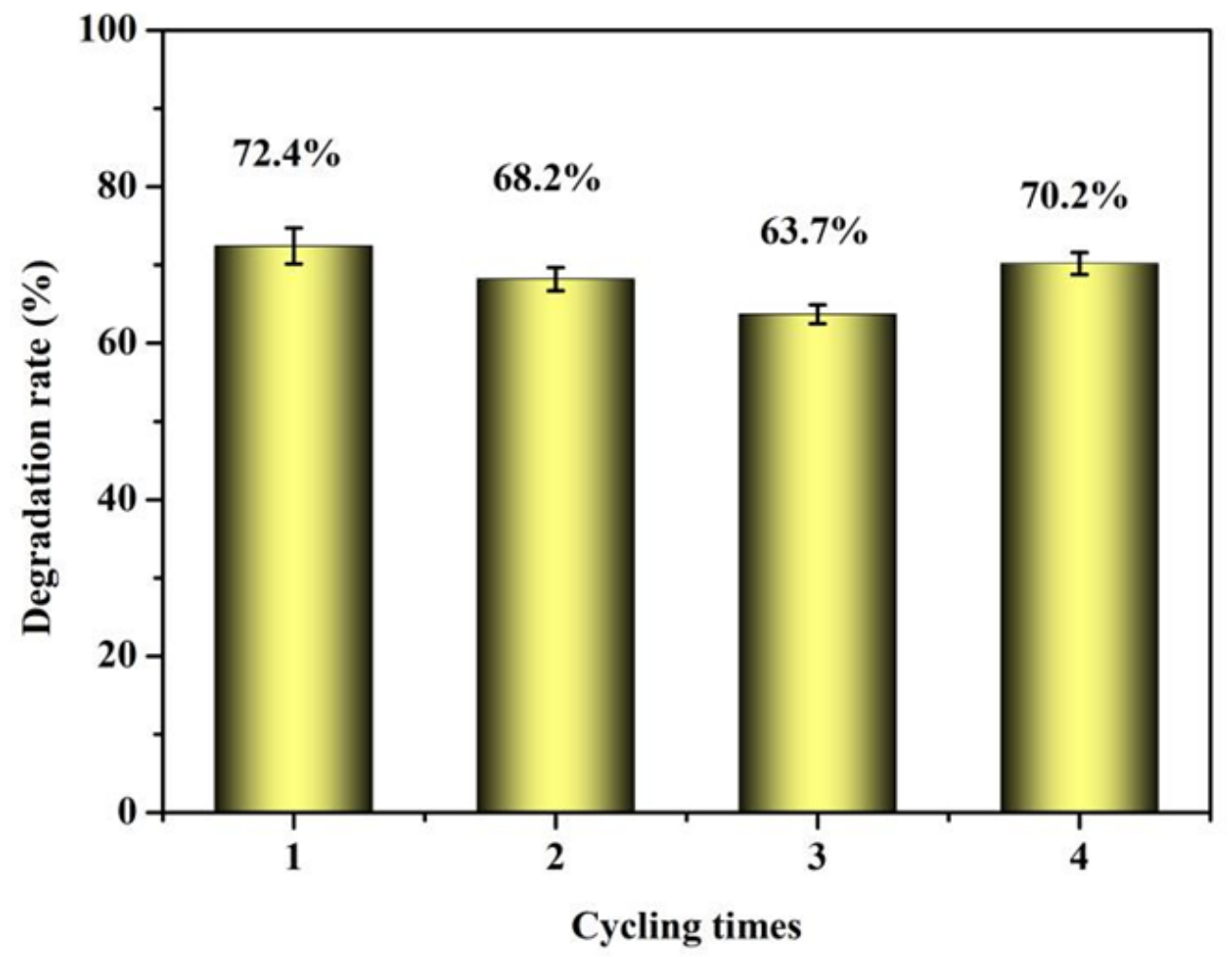

Figure 5

Reusability of the $\mathrm{Tb}, \mathrm{S}$ co-doped $\mathrm{TiO}_{2}$ composites for the degradation of MB.

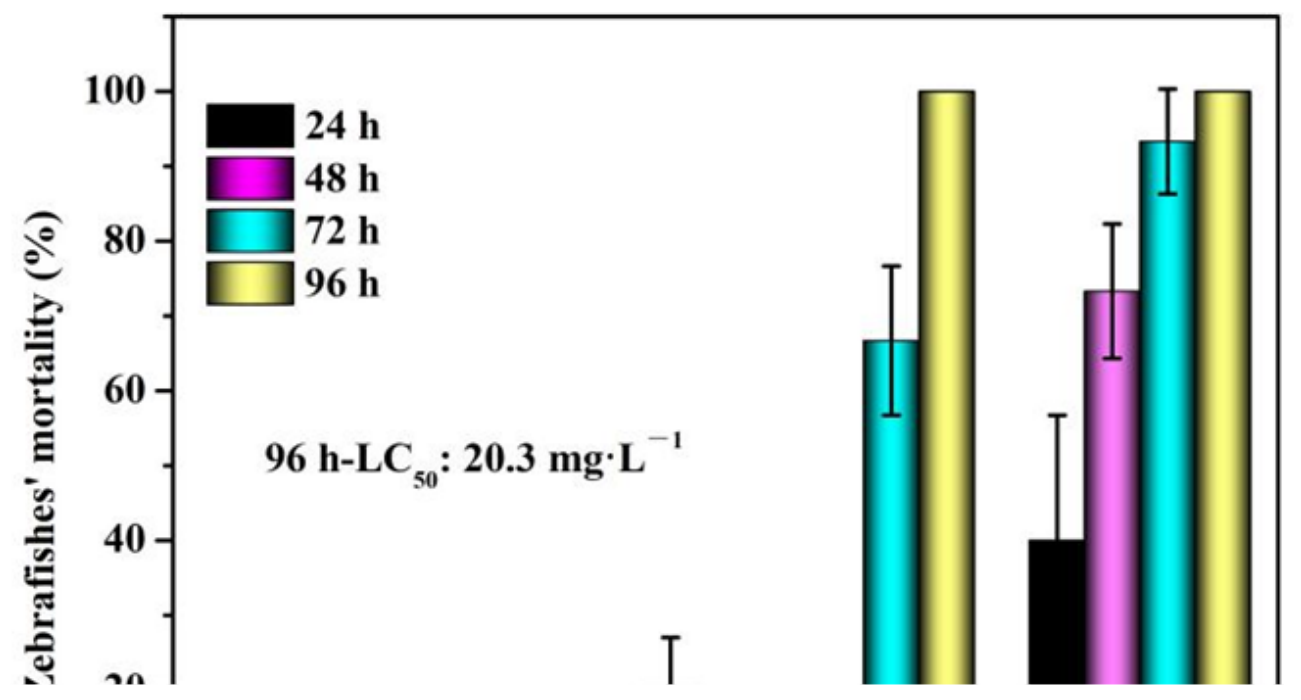

Figure 6

Mortality of zebrafish with different catalyst concentrations. 